University of Nebraska - Lincoln

DigitalCommons@University of Nebraska - Lincoln

\title{
Analysis of the Effects of the Residual Charge and Gap Size on Electrospun Nanofiber Alignment in a Gap Method
}

\author{
Lihua Liu \\ University of Nebraska - Lincoln \\ Yuris A. Dzenis \\ University of Nebraska - Lincoln, ydzenis@unl.edu
}

Follow this and additional works at: https://digitalcommons.unl.edu/engineeringmechanicsfacpub

Part of the Mechanical Engineering Commons

Liu, Lihua and Dzenis, Yuris A., "Analysis of the Effects of the Residual Charge and Gap Size on Electrospun Nanofiber Alignment in a Gap Method" (2008). Faculty Publications from the Department of Engineering Mechanics. 46.

https://digitalcommons.unl.edu/engineeringmechanicsfacpub/46

This Article is brought to you for free and open access by the Mechanical \& Materials Engineering, Department of at DigitalCommons@University of Nebraska - Lincoln. It has been accepted for inclusion in Faculty Publications from the Department of Engineering Mechanics by an authorized administrator of DigitalCommons@University of Nebraska - Lincoln. 


\title{
Analysis of the effects of the residual charge and gap size on electrospun nanofiber alignment in a gap method
}

\author{
Lihua Liu and Yuris A. Dzenis \\ Department of Engineering Mechanics, Center for Materials Research and Analysis, \\ University of Nebraska-Lincoln, Lincoln, NE 68588, USA
}

Corresponding author - L. Liu, lihualiu@unlserve.unl.edu

\begin{abstract}
In this paper, the effects of residual charges on nanofiber alignment in a gap method are studied and presented. The gap method was presented by Li and Xia (2003 Nano Lett. 3 1167); in it, a gap is introduced into a traditional collector. Due to the non-perfect conductivity of electrospun nanofibers, they carry residual charges after deposition across the gap. These residual charges will interact with the charges carried by the upcoming jet/fiber, that will also deposit across the gap. The effects of these charge interactions on nanofiber alignment were studied numerically at various gap sizes. Results showed that alignments of nanofibers improve substantially with the gap size increasing from 3 to $8 \mathrm{~mm}$. Numerical studies on the effect of residual charges in already deposited nanofibers on the alignment of nanofibers deposited afterwards were also conducted. Studies showed that the residual charges result in worse alignment, with a 10\%-25\% decrease in orientation parameters.
\end{abstract}

\section{Introduction}

Electric fields are effective and convenient to align and fabricate nanostructures, such as nanorods, nanotubes, nanowires, and nanofibers [2-7]. In electrospinning process, Huang and coauthors [8] developed a frame method to align fibers. The experimental results showed that the alignment of fibers is dependent on the frame materials. The mechanism of this method is that the special electric field induced by the frame directs and assembles the fibers into alignment. $\mathrm{Li}$ and coauthors [1] used the same principal and developed a similar and more flexible method. In their method a gap was introduced into the traditional collector, which induced a special electric field distribution that favors the alignment of nanofibers. In this method the gap size is one of the major process parameters. Various gap sizes produce different electric field distributions, which result in different degrees of nanofiber alignments. Due to the non-perfect conductivity of nanofibers, they carried with residual charges after their deposition across the gap. These residual charges interacted with the charges carried by the upcoming nanofibers in the air that are going to deposit across the gap. These charge interactions disturbed both the static electric field and the alignments of depositing nanofibers as well.

The electrified jet was modeled and represented by discrete particles with charges and mass connecting by viscoelastic elements. Dynamic system equations were developed for electrospinning process using gap alignment method. The special electric field distributions at various gap sizes were calculated through conformal transformation from regular parallel plates. Calculated electric field was submitted into the developed numerical model. Numerical simulation was employed to study the effects of residual charges on nanofiber alignment at various gap sizes. The effects of gap size on the nanofiber alignment were also simulated and validated by experimental investigation.

\section{Experimental and theoretical background}

\subsection{Experiment}

In gap alignment method, a gap was introduced into traditional substrate to collect electrospinning nanofibers 


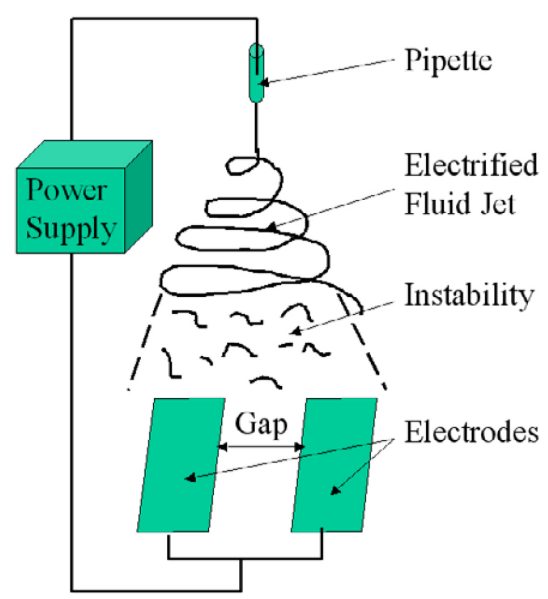

Figure 1. Schematic of gap alignment method in electrospinning process.

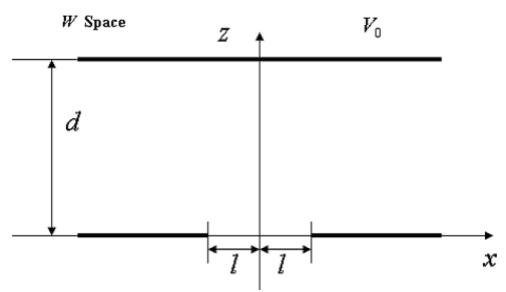

Figure 2. Electrode configuration used in gap alignment method.

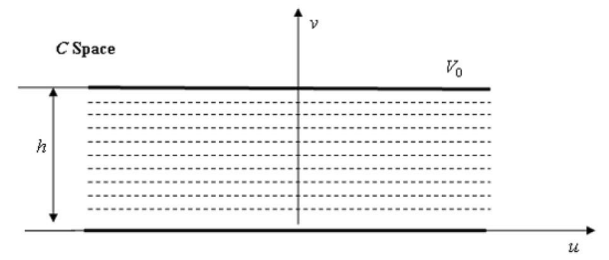

Figure 3. Equipotential lines in the image parallel-plate problem configuration.

across the gap, as illustrated in figure 1. A high voltage potential was applied between the pipette and the substrate. The inner diameter of the pipette tip was around $0.5-1.00 \mathrm{~mm}$. Polymer solution was held in the pipette, and formed a fluid drop at the tip of pipette under balance of fluid surface tension, gravity, and electric force. Once the applied electric potential increased to a critical value and overcame the surface tension, a fluid jet emitted out from the pipette, and kept stable into some distance during its traveling to the substrate. Then the fluid jet followed an expanding spiral path due to fluid jet bending instability. At the same time, the fluid jet underwent stretching, evaporation, and solidification, which formed continuous fibers with diameters in nanoscale. The presence of the gap induced a special electric field distribution, which directed and guided the deposition of nanofibers along the same direction of electric field.

High voltage potential $12 \mathrm{kV}$ was applied between the syringe and the collector by a HP E3611A DC and Gamma HV UC5-30P power supply. The distance be-

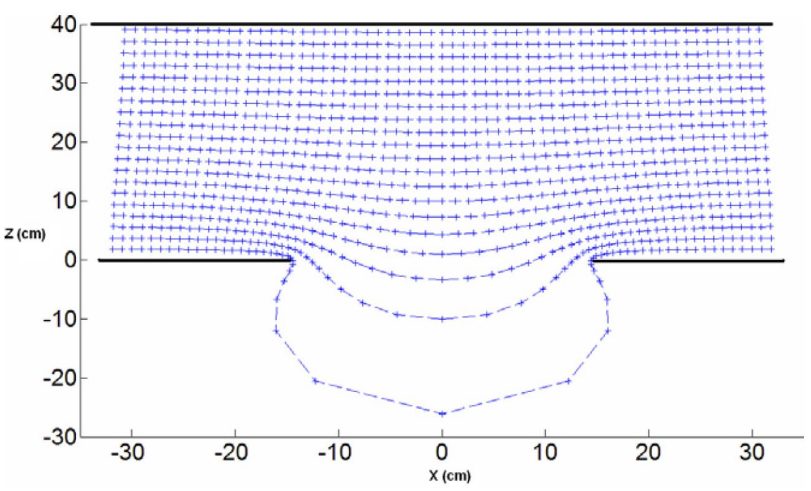

Figure 4. Calculated equipotential lines for the electrode configuration shown in figure 2 .

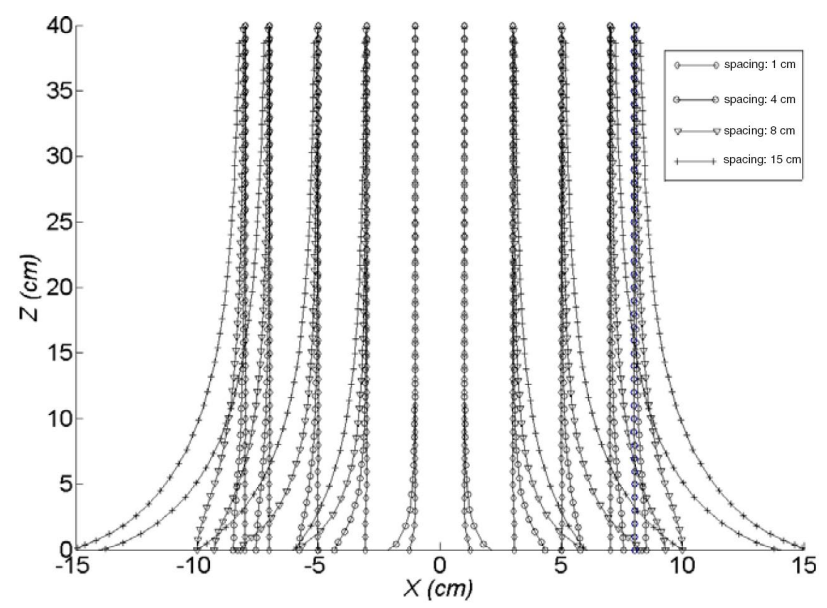

Figure 5. Calculated electric field lines in gap method for several gap sizes: $1,2,4$, and $15 \mathrm{~cm}$.

tween the syringe and collector was $40 \mathrm{~cm}$. The $4.5 \mathrm{wt} \%$ PEO solution was used. The inner diameter of the tip was $0.5-1.0 \mathrm{~mm}$. The split electrode was made from two $6 \mathrm{~cm} \times 10 \mathrm{~cm}$ metal sheets that were grounded. The gap between the two electrodes was changeable. The electrospun nanofibers were collected across the gap. To study the effect of gap size, nanofibers were collected for gap sizes $3,5,8,12,15$, and $18 \mathrm{~cm}$. The deposited nanofiber webs were extracted and cut from the gap. The sample was investigated using SEM.

\subsection{Theoretical background}

The electrified fluid jet was simply modeled as discrete particles carrying charges and mass and connected by viscoelastic elements $[9,10]$. The deposition analysis model was used to simulate the method and study the effects of residual charges and gap sizes on nanofiber alignments. The dynamic, viscoelastic, and mass conservation equations were derived in a separate paper [10] and summarized as:

$$
\frac{\mathrm{d} \sigma_{u i}}{\mathrm{~d} t}=G \frac{1}{l_{u i}} \frac{\mathrm{d} l_{u i}}{\mathrm{~d} t}-\frac{G}{\mu} \sigma_{u i},
$$




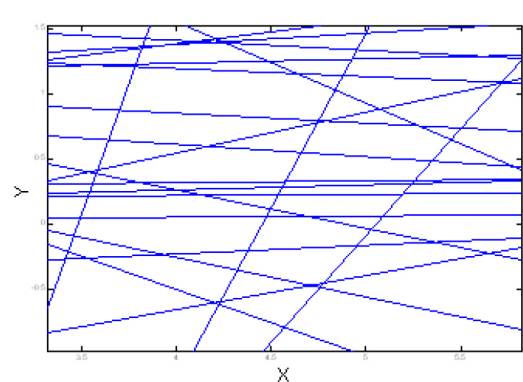

(a)

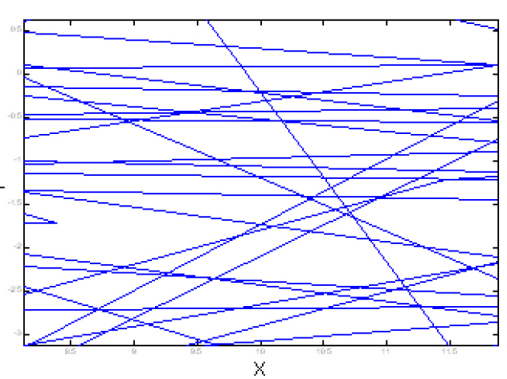

(b)

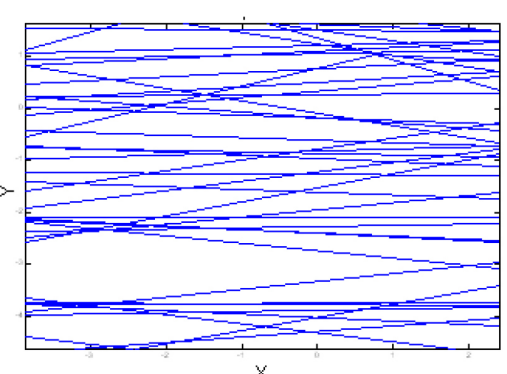

(c)
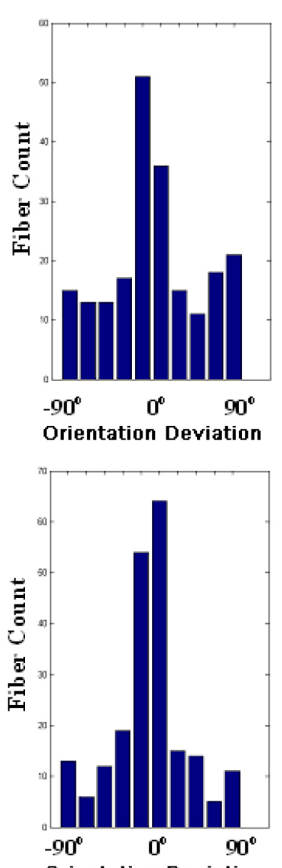

Orientation Deviation

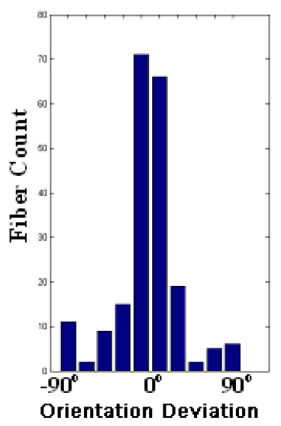

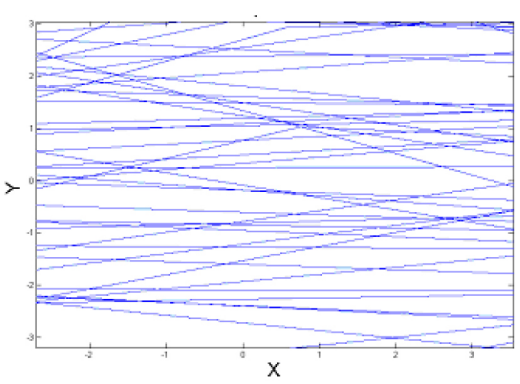

(d)

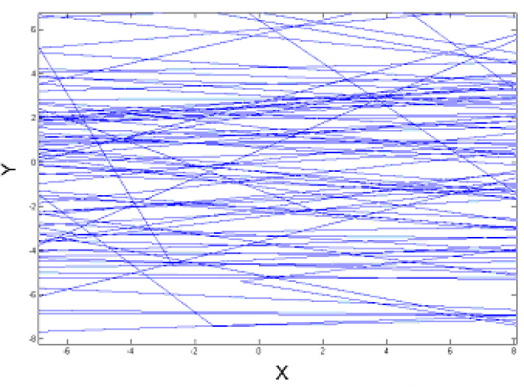

(e)

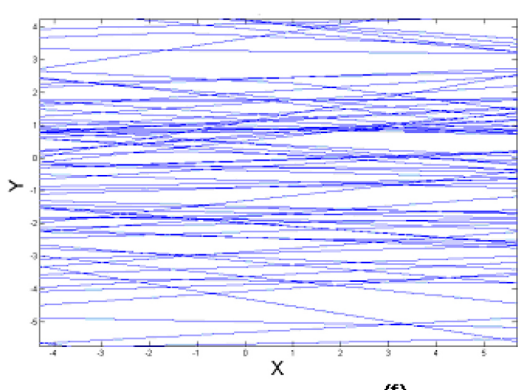

(f)
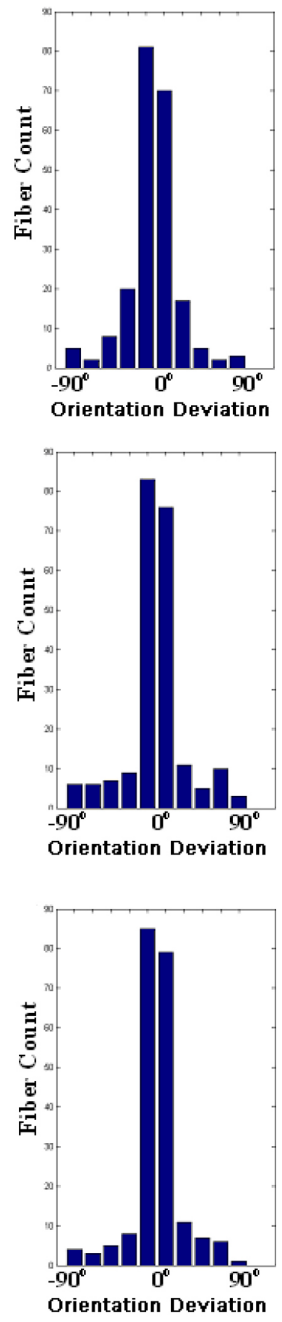

Figure 6. Simulated alignments of nanofibers for gap sizes: (a) $3 \mathrm{~cm}$; (b) $5 \mathrm{~cm}$; (c) $8 \mathrm{~cm}$; (d) $12 \mathrm{~cm}$; (e) $15 \mathrm{~cm}$; and (f) $18 \mathrm{~cm}$.

$$
\begin{gathered}
\frac{\mathrm{d} \sigma_{d i}}{\mathrm{~d} t}=G \frac{1}{l_{d i}} \frac{\mathrm{d} l_{d i}}{\mathrm{~d} t}-\frac{G}{\mu} \sigma_{d i}, \\
\pi a_{u i}^{2} l_{u i}=\pi a_{0}^{2} L, \\
\pi a_{d i}^{2} l_{d i}=\pi a_{0}^{2} L,
\end{gathered}
$$

where $a_{u i}$ and $l_{u i}$ are respectively the radius and length of the connecting element between the $i$ th and the $(i+$ 1)th particles, $a_{d i}$ and $l_{d i}$ respectively are the radius and length of the connecting element between the $i$ th and the $(i-1)$ th particles, $L$ is the initial length, $a_{0}$ is the initial cross-sectional radius at $t=0 . \sigma_{u i}$ and $\sigma_{d i}$ are the viscoelastic stresses, $t$ is time, and $G$ and $\mu$ are the elastic modulus and viscosity, respectively.

The dynamic equations are

$$
\begin{gathered}
m \frac{\mathrm{d}^{2} x_{i}}{\mathrm{~d} t^{2}}=\sum_{\substack{j=1, N \\
j \neq i}} \frac{e^{2}}{R_{i j}^{2}} \frac{x_{i}-x_{j}}{R_{i j}}+\frac{\pi a_{u i}^{2} \sigma_{u i}}{l_{u i}}\left(x_{i+1}-x_{i}\right) \\
-\frac{\pi a_{d i}^{2} \sigma_{d i}}{l_{d i}}\left(x_{i}-x_{i-1}\right) \\
-\frac{\alpha \pi\left(a^{2}\right)_{a v} k_{i}}{\left(x_{i}^{2}+y_{i}^{2}\right)^{1 / 2}}\left[\left|x_{i}\right| \operatorname{sgn}\left(x_{i}\right)\right]+e E_{x}
\end{gathered}
$$

$$
\begin{aligned}
m \frac{\mathrm{d}^{2} y_{i}}{\mathrm{~d} t^{2}} & =\sum_{\substack{j=1, N \\
j \neq i}} \frac{e^{2}}{R_{i j}^{2}} \frac{y_{i}-y_{j}}{R_{i j}}+\frac{\pi a_{u i}^{2} \sigma_{u i}}{l_{u i}}\left(y_{i+1}-y_{i}\right) \\
& -\frac{\pi a_{d i}^{2} \sigma_{d i}}{l_{d i}}\left(y_{i}-y_{i-1}\right)-\frac{\alpha \pi\left(a^{2}\right)_{a v} k_{i}}{\left(x_{i}^{2}+y_{i}^{2}\right)^{1 / 2}}\left[\left|y_{i}\right| \operatorname{sgn}\left(y_{i}\right)\right],
\end{aligned}
$$

$$
\begin{gathered}
m \frac{\mathrm{d}^{2} z_{i}}{\mathrm{~d} t^{2}}=\sum_{\substack{j=1, N \\
j \neq i}} \frac{e^{2}}{R_{i j}^{2}} \frac{z_{i}-z_{j}}{R_{i j}}+\frac{\pi a_{u i}^{2} \sigma_{u i}}{l_{u i}}\left(z_{i+1}-z_{i}\right) \\
-\frac{\pi a_{d i}^{2} \sigma_{d i}}{l_{d i}}\left(z_{i}-z_{i-1}\right)+e E_{z},
\end{gathered}
$$

where $m$ and $e$ are the mass and charge of one particle, respectively. $N$ is total number of particles. $R_{i j}$ is the distance between particle $i$ and $j ; x_{i}, y_{i}$, and $z_{i}$ are the Cartesian coordinates of the particle $i, \alpha$ is the surface tension coefficient, $k_{i}$ is the jet curvature, and $E_{x}$ and $E_{z}$ are the electric field in $x$ and $z$ direction respectively.

Parameters studies were conducted for effects of the residual charges and gap size on the orientation of deposited nanofibers. In this numerical simulation, the parameters 


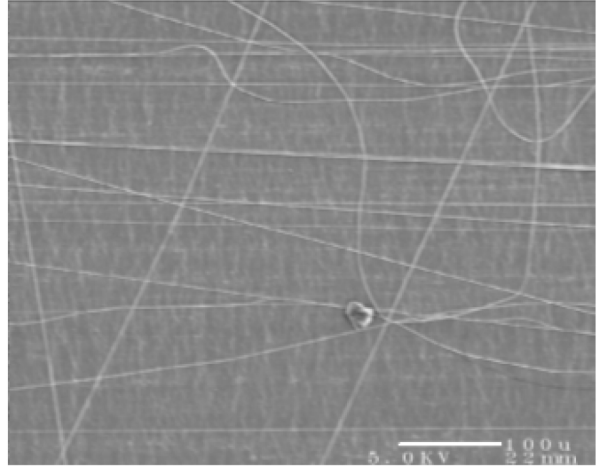

(a)

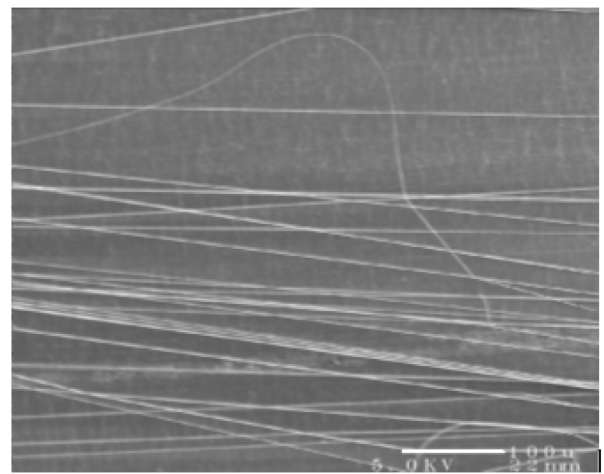

(b)

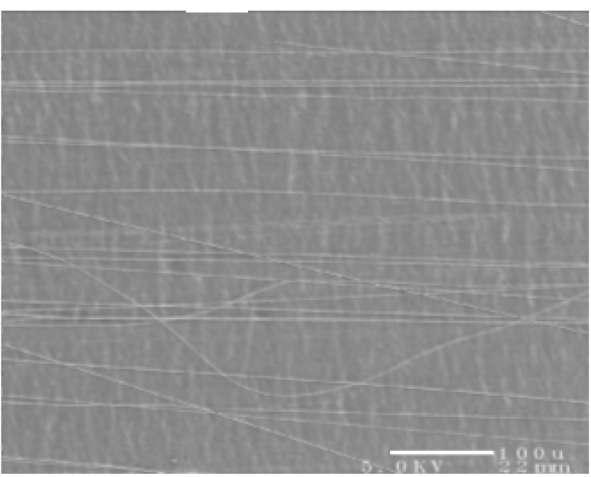

(c)

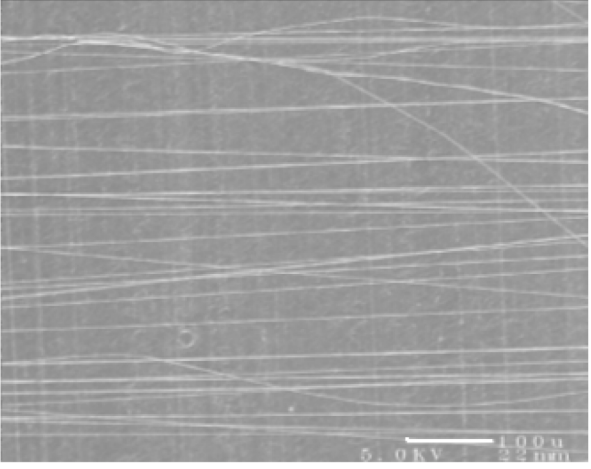

(d)

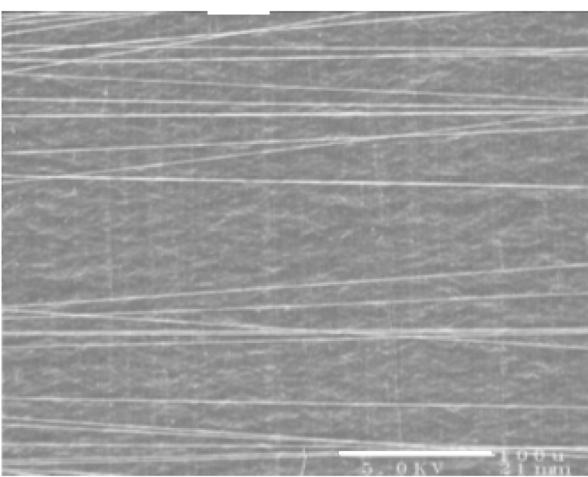

(e)

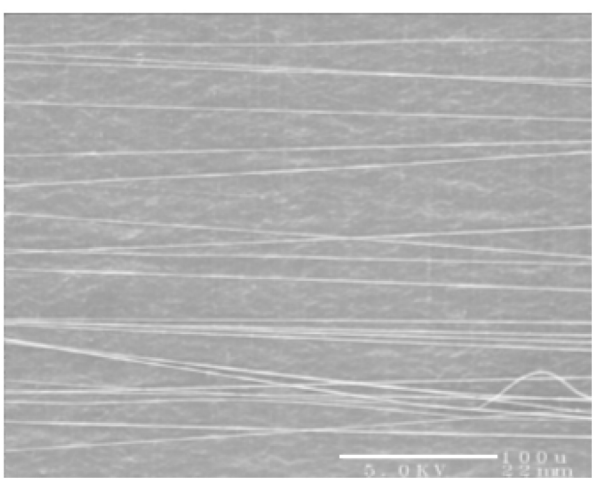

(f)

Figure 7. Observed alignments of nanofibers for gap sizes: (a) $3 \mathrm{~cm}$; (b) $5 \mathrm{~cm}$; (c) $8 \mathrm{~cm}$; (d) $12 \mathrm{~cm}$; (e) $15 \mathrm{~cm}$; and (f) $18 \mathrm{~cm}$.

were set correspondingly with the experiments described in the following part. The DC voltage $V_{0}$ is 12 $\mathrm{kV}$, distance between the tip of syringe to the collector $h$ is $40 \mathrm{~cm}$, and gap size varies from 2 to $18 \mathrm{~cm}$. The properties for the polymer solution are listed as: the initial radius of the jet $a_{0}$ is $150 \mu \mathrm{m}$, initial length $L$ is $3.19 \mathrm{~mm}$, density $\rho$ is $10^{3} \mathrm{kgm}^{-3}, \alpha$ is $0.7 \mathrm{~kg} \mathrm{~s}^{-2}, \mu$ is $10^{3} \mathrm{~kg} \mathrm{~m}^{-1} \mathrm{~s}^{-}$ 1 , relaxation time $\theta$ is $10 \mathrm{~ms}, G=\theta \cdot \mu$, the estimated jet charge density is $1 \mathrm{Cl}^{-1}$, so $e$ is $2.83 \times 10^{-9} \mathrm{C}$.

\section{Results and discussion}

\subsection{Calculation of electric field}

Introduction of gap into the traditional continuous collector changes the electric field, particularly in the vicinity of the collector. To simulate nanofiber deposition in this method, the electric field distribution needs to be calculated. Conformal transformation is used to transform the electrode configuration in Figure 2 into a simpler one in Figure 3. The length of the gap in Figure 2 is $2 l$. The distance between the upper and bottom plates is $d$. The electric equipotential lines between the parallel plates in the image problem are shown in Figure 3. The potentials of the upper and bottom plates are $V_{0}$ and 0 respectively. The distance between the two plates is $h$.

The transformation used is

$$
w=c+l^{2} / 4 c
$$




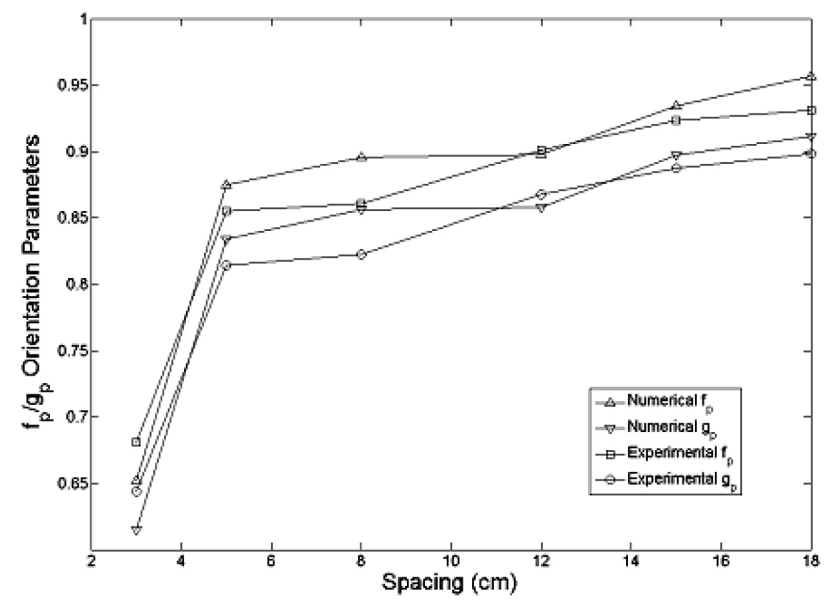

Figure 8. Variation of nanofiber orientation parameters with gap size.

where

$$
\begin{aligned}
& c=u+\mathrm{i} v, \\
& w=x+\mathrm{i} z .
\end{aligned}
$$

Separating the real and imaginary parts we obtain

$$
\begin{aligned}
& x=u+\frac{l^{2} u}{4\left(u^{2}+v^{2}\right)}, \\
& z=v-\frac{l^{2} v}{4\left(u^{2}+v^{2}\right)} .
\end{aligned}
$$

From the above equations, we obtain the equation of curves in the $w$ plane

$$
\frac{x}{u}+\frac{z}{v}=2
$$

Figure 4 shows the calculated equipotential lines in the original $w$ space. Let $u=0, v=h$. From Equation (6) we obtain

$$
d=h-l^{2} / 4 h .
$$

In general, the electric field components in the $x$ and $z$ directions are

$$
\begin{aligned}
& E_{x}=\frac{\partial V}{\partial x}=\frac{\partial V}{\partial u} \frac{\partial u}{\partial x}+\frac{\partial V}{\partial v} \frac{\partial v}{\partial x}, \\
& E_{z}=\frac{\partial V}{\partial z}=\frac{\partial V}{\partial u} \frac{\partial u}{\partial z}+\frac{\partial V}{\partial v} \frac{\partial v}{\partial z} .
\end{aligned}
$$

However, $\partial V / \partial u=0$ for parallel flat plates. Equations (9) are then simplified to

$$
\begin{aligned}
& E_{x}=\left.\frac{\partial V}{\partial x}\right|_{z}=\left.\frac{\partial V}{\partial v} \frac{\partial v}{\partial x}\right|_{z}, \\
& E_{z}=\left.\frac{\partial V}{\partial z}\right|_{x}=\left.\frac{\partial V}{\partial v} \frac{\partial v}{\partial z}\right|_{x} .
\end{aligned}
$$

By differentiating equation (6) with respect to $x$ and $z$, the electric field components are obtained as

$$
\begin{aligned}
& E_{x}=\frac{-V_{0}}{h} \\
& \times \frac{2 u z(x-u)}{16 u^{3}-24 x u^{2}+2 u\left(5 x^{2}+z^{2}+l^{2}\right)-x l^{2}-x z^{2}-x^{3}}, \\
& E_{z}=\frac{V_{0}}{h} \\
& \times \frac{16 v^{3}-20 v^{2} z+2 v\left(3 z^{2}+x^{2}-l^{2}\right)+z l^{2}}{32 v^{3}-48 v^{2} z+4 v\left(5 z^{2}+x^{2}-l^{2}\right)+2 z l^{2}-2 x^{2} z-2 z^{3}} .
\end{aligned}
$$

From equation (4), we obtain

$$
c=\frac{w \pm \sqrt{w^{2}-l^{2}}}{2} .
$$

Variables $u$ and $v$ can be expressed as functions of $x$ and $z$, which provides the electric field components only in terms of $x$ and $z$. Figure 5 illustrates the calculated electric field distribution for the following parameters: distance between the two plates $d=40 \mathrm{~cm}$, gap sizes $2 l$ are $1,4,8$, and $15 \mathrm{~cm}$, and electric potential $V=16 \mathrm{kV}$.

\subsection{Effects of gap size on nanofiber alignment}

As shown in figure 5, the electric lines are different for different spacing sizes. With gap size increasing, the electric field component in the horizontal direction also increases, which favors the alignment of nanofibers. Parametric studies of nanofiber alignment for different gap sizes were conducted. Simulations of nanofiber deposition were run for gap sizes $3,5,8,12,15$, and $18 \mathrm{~cm}$. The simulated nanofiber deposits were analyzed and nanofiber orientation histograms were calculated. The results are presented in Figure 6. The results show that nanofiber orientation was quite poor for small gap sizes. The degree of nanofiber alignment increased with the increasing of gap size. However, in all cases, the calculated nanofiber orientation distributions were broad, with some nanofibers oriented at wide angles with respect to the preferred orientation direction. These severely misoriented nanofibers may be detrimental for advanced applications.

To validate numerical simulations, experimental investigation of gap size effects on alignment was conducted. A small portion of the fibers cut from the gap was studied with SEM to determine the orientation and alignment. SEM images of obtained electrospun nanofiber assemblies are presented in Figure 7. The two electrodes were separated by the gap along the horizontal direction in this figure. The results show that the orientation was quite poor when the gap size was smaller than $5 \mathrm{~cm}$. With the gap size increased to $12 \mathrm{~cm}$, relatively well-oriented nanofibers were deposited across the gap.

The orientation of fibers in a plane can be described by fiber orientation distribution function $\Psi(\varphi)$. This function is defined such that the probability, $P$, of finding a fiber between $\varphi_{1}$ and $\varphi_{2}$ is given by

$$
P\left(\phi_{1} \leqslant \phi \leqslant \phi_{2}\right)=\int_{\phi_{1}}^{\phi_{2}} \Psi(\phi) \mathrm{d} \phi .
$$



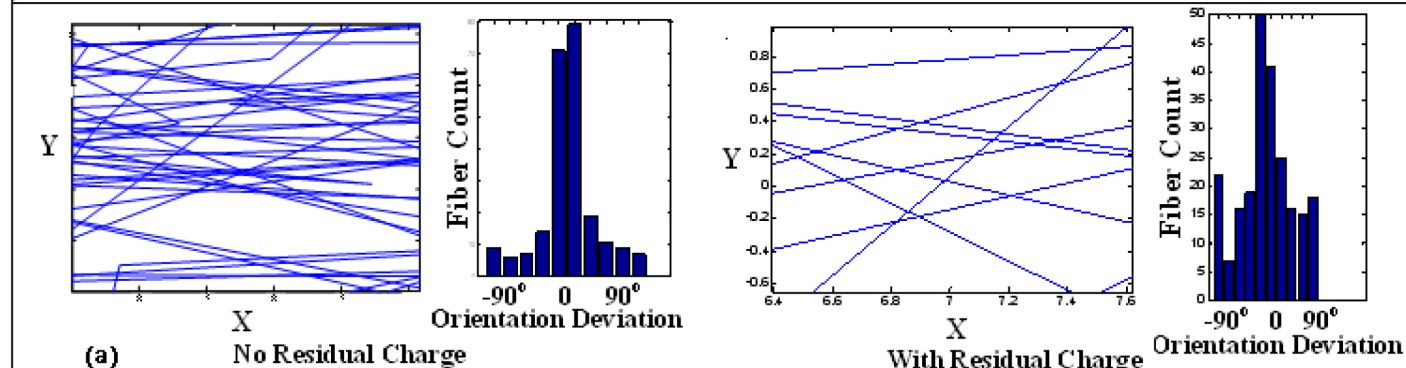

(a) No Residual Charge

With Residual Charge
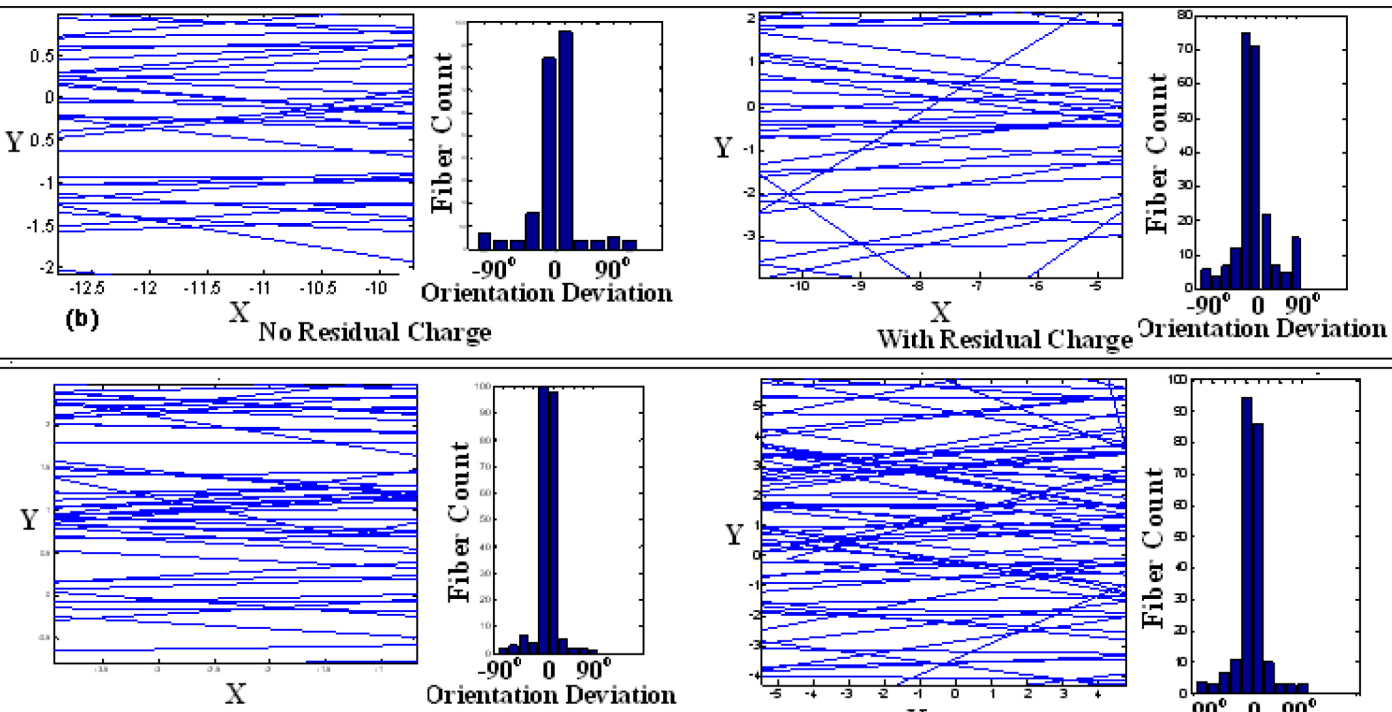

(c) No Residual Charge

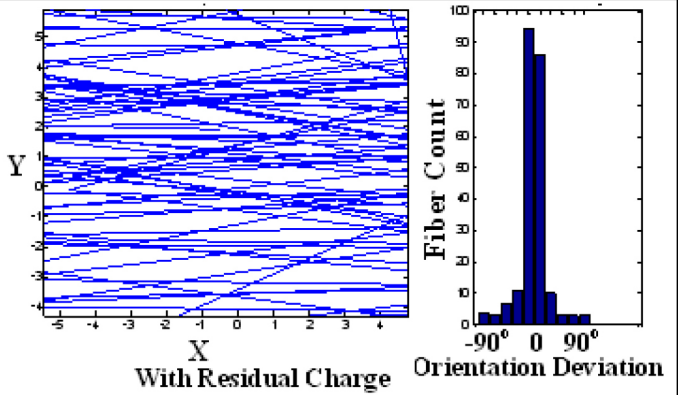

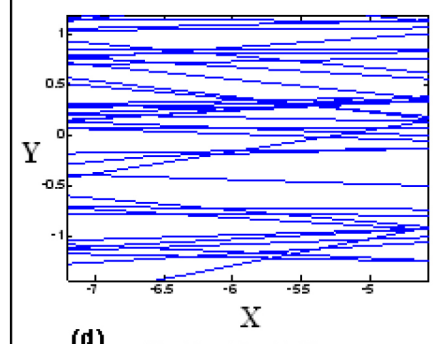

(d)
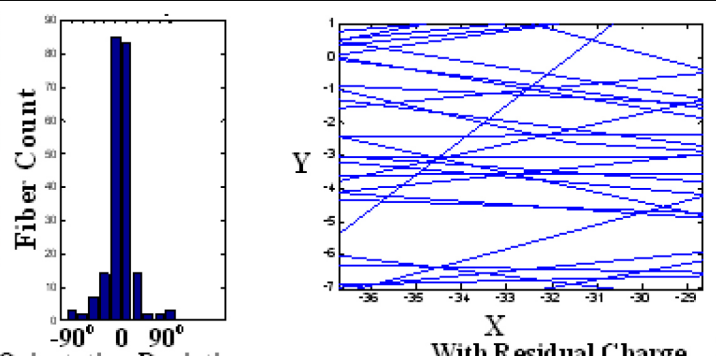

With Residual Charge

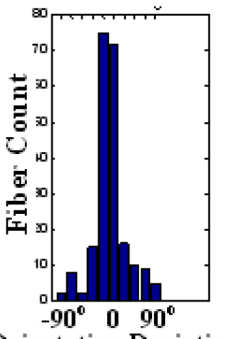

Orientation Deviation
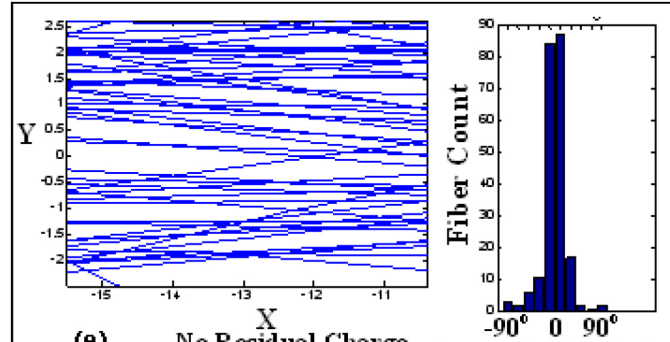

Orientation Deviation
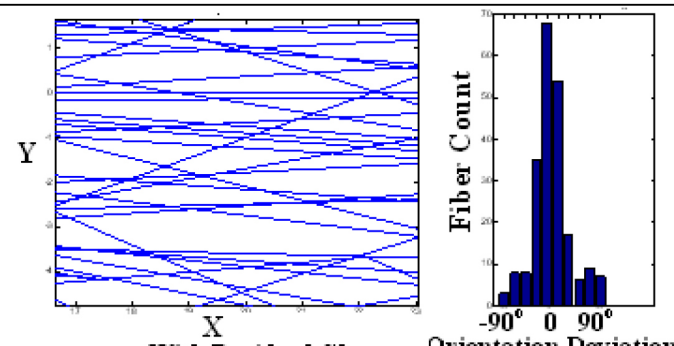

With Residual Charge Orientation Deviation

Figure 10. Effect of residual charges on the alignment of nanofiber assembly across gap.

In order to describe the orientation of nanofibers, two orientation parameters were introduced [11]

$$
\begin{gathered}
f_{P}=2\left\langle\cos ^{2} \phi\right\rangle-1 \\
g_{P}=\left(8\left\langle\cos ^{4} \phi\right\rangle-3\right) / 5
\end{gathered}
$$

where

$$
\left\langle\cos ^{m} \phi\right\rangle=\int_{0}^{\pi} \cos ^{m} \phi \Psi(\phi) \mathrm{d} \phi, \quad m=2,4 .
$$

These orientation parameters vary from $f_{P}=g_{P}=1$ for 
perfect alignment of nanofibers to $f_{P}=g_{P}=0$ for random nanofiber distribution.

For quantitative comparison of simulations and experimental observations, the orientation parameters, $f_{P}$ and $g_{P}$, were calculated based on simulation and experimental nanofiber deposition pattern. Variations of $f_{p}$ and $g_{P}$ as function of gap size are shown in Figure 8. The results show that the simulations agree well with the experimental observations. The quality of nanofiber alignment measured by the orientation parameters $f_{P}$ and $g_{P}$ increased very fast in the gap size interval from 3 to 5 $\mathrm{cm}$. The alignment then continued to slowly improve for the gap size increasing from 5 to $18 \mathrm{~cm}$. The $18 \mathrm{~cm}$ gap was the experimental maximum for the used process configuration. The highest orientation parameters at this gap size were better than the maximum parameters achieved in the drum collection method.

\subsection{Effects of residual charges on nanofiber alignment}

Unlike nanofibers deposited on conductive substrate (electrode) that can be easily discharged, the nanofibers suspended across the gap may retain their charge (residual charge). This residual charge in the deposited nanofibers may affect the deposition of the upcoming nanofibers due to electric repulsion. It is difficult to study residual charge effect experimentally. Numerical simulations were employed to evaluate the effect of residual charges on alignment of nanofibers in the gap method. The Coulomb forces by the residual charges on the depositing particles were incorporated into the dynamic equations. Simulations were conducted for gap sizes 4, $8,10,16$, and $18 \mathrm{~cm}$ with and without residual charges. The simulation results are shown in Figure 9. The qualitative analysis of the simulated nanofiber deposits and orientation histograms in Figure 9 shows that residual charges worsen the nanofiber alignment.

Orientation parameters $f_{P}$ and $g_{P}$ were calculated and used for quantitative evaluation (Figure 10). The results show that the residual charges decrease the orientation parameters. The values of the orientation parameters $f_{P}$ and $g_{p}$, are 0.70 and 0.75 , respectively, at $4 \mathrm{~cm}$ gap without residual charges. These parameters increase sharply to 0.89 and 0.85 for the gap size $8 \mathrm{~cm}$. Then they reach a plateau with the values around 0.85 . Residual charges reduce the orientation parameters by $10-25 \%$.

It is interesting that the maximum value of the orientation parameters obtained without considering residual charges is around 0.89 . This value is still lower than 1 for perfect orientation. Residual charges further reduce the maximum achievable orientation in this method.

\section{Conclusions}

In this paper, nanofiber deposition and alignment in the gap alignment method were studied numerically and experimentally. Simulations on nanofiber de- position were conducted for various gap sizes. Orientation parameters were computed and analyzed. The results showed that the alignment increased non-linearly with the increased gap size. However, segments with poor orientation were still present even in the bestaligned samples. The maximum alignment was higher than that in the rotating drum method but substantially lower than perfect alignment. Experimental observations for different gap sizes confirmed numerical predictions. Parametric studies of the effect of residual charges showed that residual charges could substantially decrease the degree of nanofiber alignment.

\section{Acknowledgments}

This research was supported in part by grants from NSF, $\mathrm{ARO} / \mathrm{ARL}$, AFOSR, and DARPA. The authors gratefully acknowledge the support. Lihua Liu would also like to express gratefully acknowledgment to the Donald F. Othmer Fellowship from the University of Nebraska-Lincoln (2001-2003).

\section{References}

[1] Li D, Wang Y L, and Xia Y N 2003 Electrospinning of polymeric and ceramic nanofibers as uniaxially aligned arrays Nano Lett. 31167

[2] Smith P A, Nordquist C D, Jackson T N, and Mayer T S 2000 Electric-field assisted assembly and alignment of metallic nanowires Appl. Phys. Lett. 771399

[3] Chen X Q, Saito T, Yamada H, and Matsushige K 2001 Aligning single-wall carbon nanotubes with an alternating-current electric field Appl. Phys. Lett. 783714

[4] Balkus K J, Madhugiri S, and Ferraris J 2002 Electrospun nanofiber composites J. Am. Chem. Soc. 224 U448 (Abstracts of Papers)

[5] Ural A, Li Y, and Dai H 2002 Electric-field-aligned growth of single-walled carbon nanotubes on surfaces Appl. Phys. Lett. 813464

[6] Zhang Y, Chang A, Cao J, Wang Q, Kim W, and Li Y 2001 Electric-field-directed growth of single-walled carbon nanotubes Appl. Phys. Lett. 793155

[7] Harnack O, Pacholski C, Weller H, Yasuda A, and Wessels J M 2003 Rectifying behavior of electrically aligned $\mathrm{ZnO}$ nanorods Nano Lett. 31097

[8] Huang Z M, Zhang Y Z, Kotaki M, and Ramakrishna S 2003 A review on polymer nanofibers by electrospinning and their applications in nanocomposites Compos. Sci. Technol. 632223

[9] Reneker D H, Yarin A L, Fong H, and Koombhongse S 2000 Bending instability of electrically charged liquid jets of polymer solutions in electrospinning J. Appl. Phys. 874531

[10] Liu L H 2007 Studies on deposition and alignment of electrospun nanofiber assemblies Dissertation University of Nebraska-Lincoln

[11] Gadala-Maria F and Parsi F 1993 Measurement of fiber orientation in short-fiber composites using digital image processing Polym. Compos. 14126 